\title{
CARACTERIZACIÓN MOLECULAR DE CEPAS DE Listeria monocytogenes AISLADAS DEL HOSPITAL MATERNO INFANTIL SAN BARTOLOMÉ, LIMA-PERÚ
}

\author{
Molecular characterization of Listeria monocytogenes strains isolated from Hospital Materno \\ Infantil San Bartolomé, Lima-Perú
}

Miguel Villegas ${ }^{1,2}$, Amparo I Zavaleta ${ }^{2}$, Javier Soto ${ }^{1}$, Nazario Silva ${ }^{1}$

${ }^{1}$ Laboratorio de Microbiología del Hospital Materno Infantil San Bartolomé. ${ }^{2}$ Laboratorio de Biología Molecular, Facultad de Farmacia y Bioquímica, Universidad Nacional Mayor de San Marcos.

\section{RESUMEN}

La listeriosis afecta principalmente a gestantes, neonatos, ancianos y pacientes con baja respuesta inmune, entre otros; es causada por Listeria monocytogenes. En este estudio se caracterizaron molecularmente 20 cepas clínicas de L. monocytogenes aisladas a partir de 18 casos de listeriosis perinatal procedente del Hospital San Bartolomé de Lima-Perú durante los años 2001 al 2005. Las bacterias fueron caracterizadas inicialmente con pruebas bacteriológicas convencionales, tales como presencia de $\beta$-hemólisis incompleta, reacción CAMP positiva con Staphylococcus aureus y negativa con Rhodococcus equi, así como, la utilización diferencial de carbohidratos D-glucosa y L-ramnosa, pero no de D-xilosa ni D-manitol. Las cepas fueron identificadas mediante la reacción en cadena de la polimerasa (PCR) y tipificadas con la técnica del ADN Polimórfico Amplificado al Azar (RAPD) utilizando los cebadores OMP-o1, M13, M132, PJ108 y PJ118. Con OMP-o1 y PJ108 se obtuvieron seis RAPD (tipo): ocho cepas con el tipo AI, tres con cada uno de los tipos A-II, A-III y B-II, dos con tipo B-I y una con el tipo A-IV. Se concluye que, de los 18 casos de listeriosis, se aislaron 20 cepas de L. monocytogenes con seis genotipos diferentes, siendo el RAPD tipo A-I, el más frecuente con el 40\% (8/20) de las cepas estudiadas.

Palabras clave: Listeria monocytogenes, listeriosis, RAPD, genotipado.

\section{SUMMARY}

Listeriosis primarily affects pregnant women, newborns, elderly people and patients with low immune response, among others, and is caused by Listeria monocytoges. In this study we characterized molecularly 20 strains of L. monocytogenes isolated from 18 cases of perinatal listeriosis in Hospital San Bartolomé of Lima-Perú, during 2001 to 2005 years. The bacteria were characterized initially by conventional bacteriological testing, such as $\beta$-hemolysis incomplete, CAMP reaction positive with Staphylococcus aureus and negative with Rhodococcus equi and the differential utilization of carbohydrates as D-glucose and L-rhamnose, but not D-xylose and D-mannitol. Then, strains were identified by polymerase chain reaction (PCR) and typified by the technique of Random Amplified Polymorphic DNA (RAPD) primers using OMP-o1, M13, M132, PJ108 and PJ108. With primers OMP-o1 and PJ108 were obtained six RAPD patterns: eight strains with AI pattern, three with AII, AIII and BII patterns each one, two and one with BI and AIV patterns, respectively. In conclusion, from the 18 cases of listeriosis were isolated 20 strains of $L$. monocytogenes with six different genotypes being the AI RAPD pattern the most common with $40 \%(8 / 20)$ of the strains studied.

Keywords: Listeria monocytogenes, listeriosis, RAPD, genotyping.

\section{INTRODUCCIÓN}

$L$ isteria monocytogenes es una bacteria intracelular Gram negativa, que se transmite por alimentos contaminados, capaz de invadir células intestinales y viajar por vía linfática o sanguínea hasta alcanzar el hígado y bazo, donde se multiplica y disemina por el organismo, con especial tropismo hacia el cerebro y el útero grávido ${ }^{(1,2)}$. $L$. monocytogenes causa listeriosis que afecta principalmente a gestantes, neonatos, ancianos; pacientes con cáncer infectados con VIH, usuarios de corticoides y trasplantados, entre otros ${ }^{(3,4)}$.
En Perú, la listeriosis no se documenta de manera sistemática, sin embargo en otros países como Estados Unidos se estiman 12 casos de listeriosis por cien mil gestantes y 8,6 casos por 100 mil nacidos vivos, con una reducción progresiva decasos debidoa las estrictas políticas de salubridad impuestas en la industria de alimentos y por la sensibilización del tema en la población ${ }^{(5)}$. En Chile, se reportó el aumento de casos hasta 4,2 por mil gestantes en un nosocomio capitalino durante el $2008^{(6)}$.

La identificación y caracterización de microorganismos patógenos emergentes, como $L$. 
monocytogenes, permite un mejor control de las cepas circulantes en casos de brotes. Por ello, en el presente estudio se identifican y caracterizan molecularmente cepas de L. monocytogenes aisladas a partir de casos de listeriosis atendidos en el Hospital San Bartolomé de Lima-Perú, un establecimiento de salud materno infantil de referencia nacional.

\section{MATERIAL Y MÉTODOS}

\section{Casos y cepas clínicas}

En los registros médicos del Hospital San Bartolomé se han reportado 52 casos de listeriosis perinatal entre los años 2001 y 2005. Fueron excluidos 34 casos (22 por cultivos negativos, 10 por cultivos ser diferentes a Listeria y dos por falta de datos), de los 18 restantes, se aislaron 20 cepas de Listeria sp. debido a que en dos de estos casos se aislaron 2 cepas. Se revisaron los historiales clínicos correspondientes (de la madre y del recién nacido) y se registraron los datos clínicos y epidemiológicos pertinentes.

\section{Caracterización bacteriológica}

Se realizaron cultivos sobre agar sangre de carnero al $5 \%$ y agar tripticasa de soya, incubándose a $37{ }^{\circ} \mathrm{C}$ durante $48 \mathrm{~h}$ en condiciones de aerobiosis. Con las colonias obtenidas se identificó el género y especie bacteriana: el género Listeria se determinó mediante técnica s convencionales como tinción Gram, pruebas de catalasa, motilidad diferencial, rojo de metilo y Voges-Proskauer, tolerancia al $\mathrm{NaCl} 6,5 \%$ y crecimiento en agar bilis esculina; la especie $L$. monocytogenes se determinó con la presencia de $\beta$-hemólisis, prueba de CAMP (con $S$. aureus ATCC 25923 y $R$. equi ATCC 6939) y fermentación de carbohidratos: D-glucosa, L-ramnosa, D-xilosa y D-manitol.

\section{Caracterización molecular}

Extracción y purificación del ADN genómico. Se obtuvieron cultivos líquidos de L. monocytogenes en caldo tripticasa de soya con extracto de levadura al o,6\% incubados a $37^{\circ} \mathrm{C}$ por $18 \mathrm{~h}$, luego se centrifugaron a 5000 rpm por 5 min y lavaron de dos a tres veces con buffer salino fosfato. Cada precipitado celular se resuspendió en buffer Tris-NaCl-EDTA y lisozima, e incubó a $37{ }^{\circ} \mathrm{C}$ por $2 \mathrm{~h}$. Después, se agregó SDS al 1\% y proteinasa K (100 $\mu \mathrm{g} / \mathrm{mL}$ ) e incubó a $50^{\circ} \mathrm{C}$ durante $12 \mathrm{~h}$. El ADN se extrajo con igual volumen de fenol-cloroformo (1:1), se precipitó con acetato de sodio $3 \mathrm{M}$ e isopropanol y centrifugó a $1200 \mathrm{rpm}$ por $5 \mathrm{~min}$. Finalmente, el precipitado se lavó con etanol al $70 \%$, se disolvió en $50 \mu \mathrm{L}$ de buffer Tris
EDTA y se guardó a $4{ }^{\circ} \mathrm{C}$. El ADN purificado se separó por electroforesis en gel de agarosa al $1 \%$ en buffer Tris-Acetato-EDTA $1 \mathrm{X}$ a $80 \mathrm{~V}$ por 1 h. La concentración del ADN extraído se determinó por comparación con cantidades conocidas del marcador de peso molecular fago Lambda / Hind III.

Reacción en cadena de la polimerasa (PCR). Se utilizaron cebadores diseñados a partir del gen iap específicos para L. monocytogenes: Mono A (5'-CAAACTGCTAACACAGCTACT-3') y LisıB: (5'-TTATACGCGACCGAAGCCAAC-3'), según Bubert y col ${ }^{(7)}$. El volumen final de la reacción fue de $50 \mu \mathrm{L}$, conteniendo buffer $1 \mathrm{X}, \mathrm{MgCl}_{2}$ 1,5 mM, desoxirribonucleótidos trifosfato (dNTP) $200 \mu \mathrm{M}$ de cada uno, enzima Taq ADN polimerasa $1 \mathrm{U}$, cebadores

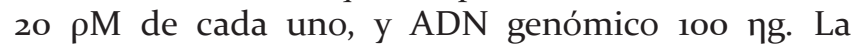
amplificación se realizo en el termociclador Perkin Elmer 2400. Las condiciones de reacción fueron las siguientes: a) desnaturalización inicial a $95{ }^{\circ} \mathrm{C}$ por 3 min; b) 35 ciclos de desnaturalización a $94{ }^{\circ} \mathrm{C}$ por $45 \mathrm{~s}$, hibridación a $55{ }^{\circ} \mathrm{C}$ por 1 min y extensión a 72 ${ }^{\circ} \mathrm{C}$ por $45 \mathrm{~s}$, c) un ciclo final de extensión de $72{ }^{\circ} \mathrm{C}$ por $7 \mathrm{~min}$. Se utilizaron controles de ADN positivo (L. monocytogenes ATCC 19118) y negativo (E. coli ATCC 43887). Los productos de PCR se separaron por electroforesis en gel de agarosa al $1 \%$ utilizando buffer Tris-Borato-EDTA $1 \mathrm{X}$ a $80 \mathrm{~V}$ durante 1 h. Se utilizó el marcador de peso molecular ladder $1 \mathrm{~kb}$.

ADN polimórfico amplificado al azar (RAPD). Se utilizaron 5 cebadores de secuencia arbitraria y tamaño variable de 10 a 20 bases descritos por otros investigadores ${ }^{(8-14)}$. Estos son: OMP-o1 (CTTGGTGGCT), M132 (GAGGGTGGCGGTTCT), PJ118 (TGTTCGTGCTGTTTCTG), PJ108 (GCTTATTCTTGACATCCA) y (GTTGTAAAACGACGGCCAGT). El volumen final de la reacción de PCR fue de $50 \mu \mathrm{L}$ conteniendo buffer $1 \mathrm{X}$, $\mathrm{MgCl}_{2} 2,5 \mathrm{mM}$, dNTP $200 \mu \mathrm{M}$ de cada uno, Taq DNA polimerasa $1 \mathrm{U}$, cebador $20 \rho \mathrm{M}$ y ADN genómico 50 ๆg. Las condiciones de amplificación fueron semejantes a las descritas antes para la PCR, excepto la temperatura de hibridación que fue de $36{ }^{\circ} \mathrm{C}$ para el cebador OMPo1 y $50{ }^{\circ} \mathrm{C}$ para el resto de cebadores. Los productos del RAPD fueron separados por electroforesis en gel de agarosa al $1 \%$, utilizando buffer TBE 1 X. Se utilizaron los marcadores de peso molecular ladder $100 \mathrm{pb}$ y 1 kb. Los perfiles de ADN se registraron con una cámara fotográfica polaroid y fueron analizados visualmente. Los fragmentos de ADN obtenidos en el RAPD se calificaron como idénticos o diferentes en base al número y tamaño de los fragmentos de ADN obtenidos para cada cebador. 
Asimismo, según los perfiles de ADN observados, las cepas en estudio se agruparon por genotipos.

\section{RESULTADOS}

Seidentificaron zocepasde L. monocytogenesaisladas a partir de 18 casos de listeriosis perinatal atendidos en el Hospital San Bartolomé de Lima (tablas 1 y 2). En el caso 4, se aisló una cepa del líquido amniótico de la madre y otra de la sangre de su recién nacido (cepas 4y 4') y en el caso 12, se aislaron dos cepas de un mismo neonato, de secreción conjuntival y sangre (cepas 12 y 12,', respectivamente). Las gestantes fueron mujeres jóvenes con edad promedio de 25 años y rango de edades entre 16 a 35 años, el 61\% (11/18) proceden de los distritos del cono norte y $39 \%$ (7/18) del cercado de Lima, incluyendo La Victoria. El $83 \%(15 / 18)$ de las madres tuvieron recién nacidos vivos, no obstante, se registraron tres casos fatales.

De las cepas en estudio, el $25 \%(5 / 20)$ se aisló de gestantes y el 75\% (15/20) de recién nacidos; $65 \%$ (13/20) a partir de muestras de sangre, $15 \%$ (3/20) de líquido amniótico, $15 \%$ (3/20) líquido cefalorraquídeo y $5 \%$ $(1 / 20)$ secreción conjuntival. F Todas las cepas estudiadas mostraron las mismas características fenotípicas de $L$. monocytogenes; fueron positivas a la prueba de catalasa, motilidad y rojo de metilo/Voges Proskauer, toleraron bilis y sal, presentaron $\beta$-hemólisis incompleta y tuvieron reacción de CAMP positiva con $S$. aureusy negativa con $R$. equi. Asimismo, tuvieron fermentación de carbohidratos diferenciada con utilización de D-glucosa y L-ramnosa, pero no de D-manitol ni D-xilosa.

La identificación molecular de las cepas se determinó mediante la visualización de los productos amplificados del gen iap de $L$. monocytogenes obtenidos por PCR, siendo el tamaño de los productos amplificados de aproximadamente $660 \mathrm{pb}$. El RAPD, con los cebadores M132, PJ118 y M13, mostró perfiles de ADN idénticos para todas las cepas. Sin embargo, con los cebadores OMP-o1 y PJ108, se obtuvieron fragmentos de $A D N$ entre o,2 y 3,o kb. En base al número y tamaño de fragmentos observados, se determinó arbitrariamente los RAPD tipo del presente estudio. Con OMP-or se obtuvieron dos perfiles de ADN diferentes denominados A y B (figura 1); mientras que con PJ108 se visualizaron cuatro perfiles de ADN asignados como I, II, III y IV (figura 2).

De las cepas clínicas en estudio, 15 (75\%) se catalogaron dentro del tipo A y 5 (25\%) del tipo B; del mismo modo, 10 fueron consideradas del tipo I, 6 del tipo II, 3 del tipo III y una del tipo IV (5\%). El cebador PJ108 permitió discriminar en 4 subgrupos las cepas
Tabla 1. Casos de listeriosis perinatal identificados en el Hospital San Bartolomé de Lima.

\begin{tabular}{|c|c|c|c|c|}
\hline \multirow[b]{2}{*}{$\begin{array}{c}\text { Caso } \\
\text { No }\end{array}$} & \multicolumn{2}{|c|}{ Gestante } & \multirow[b]{2}{*}{$\begin{array}{l}\text { Producto de } \\
\text { la gestación }\end{array}$} & \multirow[b]{2}{*}{$\begin{array}{c}\text { Fecha de } \\
\text { presentación }\end{array}$} \\
\hline & $\begin{array}{l}\text { Procedencia } \\
\text { (Distrito) }\end{array}$ & $\begin{array}{c}\text { Edad } \\
\text { (años) }\end{array}$ & & \\
\hline 1 & Comas & 19 & RN vivo & Feb 2001 \\
\hline 2 & La Victoria & 21 & RN vivo & Jul 2001 \\
\hline 3 & Cercado & 20 & RN vivo & Ago 2001 \\
\hline 4 & Cercado & 22 & RN vivo & Oct 2001 \\
\hline 5 & Cercado & 23 & RN vivo & Oct 2002 \\
\hline 6 & Puente Piedra & 26 & Óbito Fetal & Ene 2003 \\
\hline 7 & Cercado & 27 & Natimuerto & Ene 2003 \\
\hline 8 & SJL & 26 & RN vivo & Feb 2003 \\
\hline 9 & Comas & 35 & RN vivo & Oct 2003 \\
\hline 10 & Los Olivos & 26 & RN vivo & Ene 2004 \\
\hline 11 & La Victoria & 29 & Aborto & Abr 2004 \\
\hline 12 & Comas & 29 & RN vivo & Jul 2004 \\
\hline 13 & Comas & 22 & RN vivo & Nov 2004 \\
\hline 14 & Comas & 24 & RN vivo & Nov 2004 \\
\hline 15 & Cercado & 20 & RN vivo & Nov 2004 \\
\hline 16 & Independencia & 21 & RN vivo & Mar 2005 \\
\hline 17 & Los Olivos & 35 & RN vivo & May 2005 \\
\hline 18 & Comas & 16 & RN vivo & Jun 2005 \\
\hline
\end{tabular}

RN, recién nacido o neonato; SJL, San Juan de Lurigancho.

Tabla 2. Cepas de L. monocytogenes aisladas en el Hospital San Bartolomé de Lima.

\begin{tabular}{clccc}
\hline Código & \multirow{2}{*}{ MePA } & Muestra biológica & Grupo & \multicolumn{2}{c}{ RAPD tipo } \\
\cline { 3 - 4 } & Poblacional & OMP-01 & PJ108 \\
\hline 1 & Sangre & Neonato & A & II \\
2 & Sangre & Neonato & A & III \\
3 & Sangre & Neonato & A & I \\
4 & Líquido Amniótico & Gestante & A & II \\
4 & Sangre & Neonato & A & II \\
5 & LCR & Neonato & B & I \\
6 & Líquido Amniótico & Gestante & A & I \\
7 & Sangre & Neonato & A & I \\
8 & Sangre & Neonato & B & I \\
9 & Sangre & Neonato & A & I \\
10 & Sangre & Neonato & A & IV \\
11 & Sangre & Gestante & A & I \\
12 & Secreción Conjuntival & Neonato & A & III \\
12 & Sangre & Neonato & A & III \\
13 & Sangre & Neonato & B & II \\
14 & LCR & Neonato & B & II \\
15 & LCR & Neonato & B & II \\
16 & Sangre & Gestante & A & I \\
17 & Líquido Amniótico & Gestante & A & I \\
18 & Sangre & Neonato & A & I \\
\hline
\end{tabular}

LCR, líquido cefalorraquídeo.

de perfil tipo A y en dos grupos las cepas tipo B; así se obtuvieron seis RAPD tipo denominados: A-I, A-II, A-III, A-IV, B-I y B-II. El genotipo más frecuente fue el RAPD tipo A-I con el $40 \%$ de las cepas en estudio (8/20). Las cepas se distribuyeron así: ocho en el tipo A-I, tres en cada uno de los tipos A-II, A-III y B-II, dos en el tipo B-I y una en el tipo A-IV (Tabla 2). No se encontró relación entre genotipo y edad, procedencia de la madre, tipo de muestra biológica, ni grupo poblacional de aislamiento.

Se observó concordancia de genotipos en las cepas pareadas: tipo A-II para las cepas 4 y 4' de la 


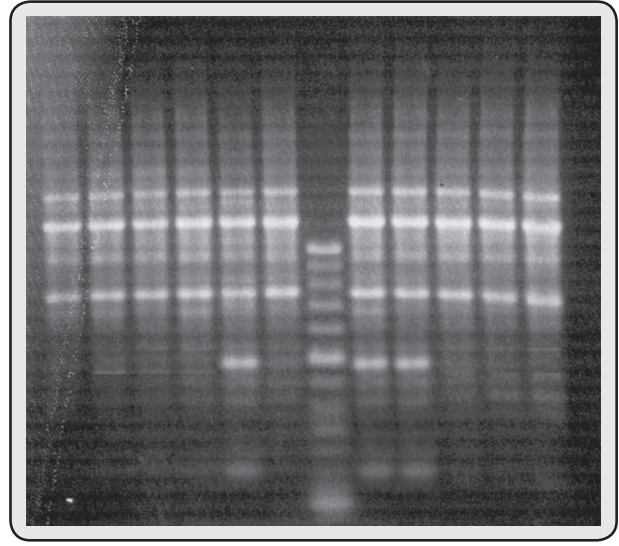

Figura 1. RAPD de las cepas de $L$. monocytogenes obtenidos con cebador OMP-01 en gel de agarosa al 1\%. El números de las líneas indican el código de cepa y las letras señalan el tipo de perfil de ADN obtenido con el cebador OMP-01; Lm, $L$. monocytogenes ATCC 19118; MP, marcador de peso molecular (ADN del fago Lambda/ Hind III).

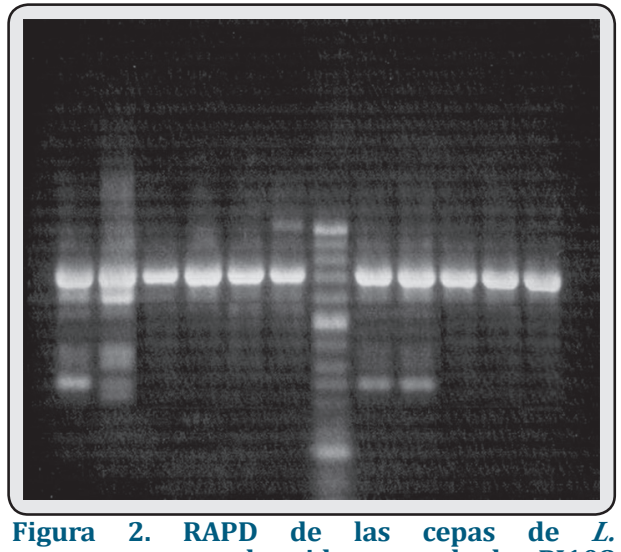

Figura 2. RAPD de las cepas de $L$.
monocytogenes obtenidos con cebador PJ108 en gel de agarosa al 1\%. Los números arábigos indican el código de cepa y los números romanos señalan el tipo de perfil de ADN obtenido con el cebador PJ108; Lm, L. monocytogenes ATCC 19118; MP, marcador de peso molecular (ADN del fago Lambda/ Hind III). madre y su neonato correspondiente, y tipo A-III para las cepas 12 y 12' de sangre y secreción conjuntival de un mismo neonato.

\section{DISCUSIÓN}

La susceptibilidad de la listeriosis en la gestación está documentada en más de 40 especies animales, incluida el hombre, siendo los factores de riesgo para desarrollar infección: la disminución de la inmunidad y la permeabilidad de la barrera placentaria ${ }^{3,15)}$. La transmisión perinatal puede ocurrir por vía transplacentaria en cualquier momento del embarazo, durante el parto (por aspiración o deglución de secreciones contaminadas) o después del parto por transmisión cruzada o ingesta de leche contaminada ${ }^{(16)}$. Se considera que, si la gestante está infectada, entonces el feto será afectado en más del 90\% de los casos, conllevando al aborto, óbito fetal o parto prematuro con sepsis neonatal ${ }^{(17)}$. La enfermedad materna es benigna pero la afectación del producto es grave y letal en el 20 a $30 \%$ de los casos ${ }^{(18)}$, en este estudio se encontró pérdida del producto en el $17 \%$ de casos.

Elaislamientobacterianodemuestras noestériles como heces, meconio y secreción cervicovaginal, no tienen mayor significado sin un contexto clínico compatible; así, por ejemplo, seaísla L. monocytogenes en las heces del 1 al 10\% de personas sanas ${ }^{(19)}$. En este trabajo, las muestras clínicas procedieron de lugares estériles, principalmente de sangre (65\%). Durante la gestación, el estado de portador asintomático en secreción cervicovaginal representa un factor de riesgo para la transmisión al producto, vía canal del parto, pero no para el desarrollo de enfermedad en la madre ${ }^{(20)}$.

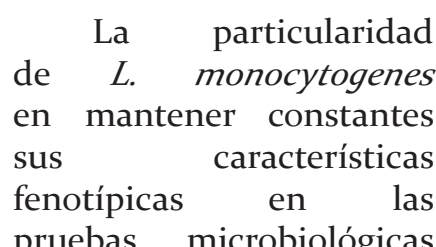
pruebas microbiológicas convencionales, hace necesaria la utilización de técnicas moleculares para su tipificación, así como para determinar asociación entre cepas aisladas e infección ${ }^{(21)}$. Las pruebas serológicas no ayudan en el ámbito clínico porque unos pocos serotipos (4b, 1/2a y $1 / 2 b)$ se asocian al $95 \%$ de todas las infecciones ${ }^{(22)}$. El RAPD es una técnica molecular sencilla, rápida y de bajo costo, que permite discriminar eficazmente entre cepas no relacionadas, aunque se requiere estandarizar previamente las condiciones de la PCR para garantizar su reproducibilidad ${ }^{(23)}$.

Lawrence y col. ${ }^{(9)}$, utilizando el cebador OMP-o1 identificaron 33 RAPD tipo de 91 cepas no relacionadas de $L$. monocytogenes de origen ambiental, alimentario y clínico. En otro estudio, Gravesen y col. ${ }^{(10)}$, compararon diferentes técnicas de genotipificación, observando 10 RAPD tipo en 48 cepas de $L$. monocytogenes de origen animal y ambiental, con capacidad discriminatoria moderada de la técnica. No obstante, los investigadores precisanquenoexistecorrelaciónentrelostiposobtenidos por las diferentes técnicas. Wagner y col. ${ }^{(11)}$, empleando la técnica RAPD con 20 cebadores, identificaron 10 RAPD tipo en 52 cepas de $L$. monocytogenes procedentes de una fábrica de alimentos, sin embargo, cuando utilizaron el cebador OMP-o1 identificaron solo 6 RAPD tipo.

Lado y Yousef ${ }^{(13)}$, compararon la tipificación molecular de 9 cepas de $L$. monocytogenes mediante las técnicas RAPD y PFGE. Con la técnica RAPD, utilizaron los cebadores PJ108 y PJ118, identificando 3 RAPD tipo; mientras que con PFGE observaron 9 tipos. Noobstante, Louie y col ${ }^{(14)}$ estudiaron 51 cepas de L. monocytogenes mediante las técnicas RAPD, ribotipificación y PFGE; observaron 21 patrones de banda con la técnica RAPD, siendo la más rápida, sencilla, barata y de alto poder discriminatorio. En esta investigación, se encontró mejor capacidad discriminatoria con el cebador PJ108 
que con el empleo de OMP-o1, semejante a lo señalado por otros autores ${ }^{(13,14)}$.

En conclusión, se aislaron 20 cepas clínicas de L. monocytogenes a partir de 18 casos de listeriosis perinatal atendidos en el Hospital San Bartolomé de Lima, todas las cepas se identificaron mediante las características fenotípicas convencionales y se confirmaron por PCR. Las cepas en estudio, se tipificaron mediante la técnica RAPD con los cebadores OMP-o1 y PJ108, observando 6 perfiles RAPD tipo: 8 cepas del tipo A-I, 3 en cada uno de los tipos A-II, A-III y B-II, 2 del tipo B-I y una del tipo IV.

\section{REFERENCIAS BIBLIOGRAFICAS}

1. Benadof D. Listeria monocytogenes. Rev Chilena Infectol 2008; 25(5): 350 .

2. Miranda G, Orellana $P$, Dellien $H$, Switt M. Romboencefalitis por Listeria monocytogenes. Patología emergente en relación al brote epidémico. Presentación de tres casos clínicos. Rev Med Chile 2009; 137(12): 1602-6.

3. Ramaswamy V, Cresence V, Rejitha J, Lekshmi M, Dharsana $\mathrm{K}$, Prasad S, et al. Listeria-review of epidemiology and pathogenesis. J Microbiol Immunol Infect 2007; 40(1): 4-13.

4. Freitag N, Port G, Miner M. Listeria monocytogenes-from saprophyte to intracellular pathogen. Nat Rev Microbiol 2009; 7(9): 623-8.

5. Janakiraman V. Listeriosis in pregnancy: diagnostic, treatment, and prevention. Rev Obstet Gynecol 2008; 1(4): 179-85.

6. Noriega M, Ibáñez S, González P, Yamamoto M, Astudillo J, González M, et al. Listeria monocytogenes: Informe de un aumento de casos en mujeres embarazadas y revisión de la literatura. Rev Chilena Infectol 2008; 25(5): 342-9.

7. Bubert A, Hein I, Rauch M, Lehner A, Yoon B, Goebel W, Wagner M. Detection and differentiation of Listeria spp. by single reaction based on multiplex PCR. Appl Environ Microbiol 1999; 65(10): 4688-92.

8. Estrada O, Zavaleta A. Genotipificación de cepas Listeria monocytogenes aisladas de leche cruda de diferentes ganaderías de Lima mediante el ADN polimórfico amplificado al azar. Ciencia e Investigación 2004; 7(1): 9-15.

9. Lawrence L, Harvey J, Gilmour A. Development of random amplification of Polymorphic DNA typing method for Listeria monocytogenes. Appl Environ Microbiol 1993; 59(9):3117-9.

10. Gravesen A, Jacobsen T, Moller P, Hansen F, Larsen A, Kochel S. Genotyping of Listeria monocytogenes: comparison of RAPD, ITS and PFGE. Int J Food Microbiol 2000; 57(1-2): 43-51.

11. Wagner M, Maderner A, Brandl E. Random amplification of polymorphic DNA for tracing and molecular epidemiology of Listeria contamination in cheese plant. J Food Protection 1996; 59(6): 384-9.

12. Wulf G, Gram L, Ahrens P, Vogel BF. One group of genetically similar Listeria monocytogenes strains frequently dominates and persists in several fish slaughter and smokehouses. App Environ Microbiol 20o6; 72(6): 4313-22.

13. Lado B, Yousef A. Selection and identification of a Listeria monocytogenes target strain for pulsed electric field process optimization. App Environ Microbiol 2003; 69(4): 2223-9.

14. Louie M, Jayaratne P, Luchsinger I, Devenish J, Yao J, Schlech W, et al. Comparison of ribotyping, arbitrarily primed PCR and pulsed-field gel electrophoresis for molecular typing of Listeria monocytogenes. J Clin Microbiol 1996; 34(1): 15-9.

15. Larraín D, Abarzúa F, De Jourdan F, Merino P, Belmar C, García P. Infecciones por Listeria monocytogenes en mujeres embarazadas: experiencia del Hospital Clínico de la Pontificia Universidad Católica de Chile. Rev Chilena Infectol 2008; 25(5): 336-41.

16. Robbins J, Skrzypczynska K, Zeldovich V, Kapidzid M, Bakardjiev A. Placental syncytiotrophoblast constitutes a major barrier to vertical transmission of Listeria monocytogenes. PLoS Pathog 2010; 6(1): e1000732.

17. Costa M, Toyos P, Coto G, Fernández B, López J. Listeriosis perinatal: ¿una infección emergente? An Pediatr (Barc) 2009; 70: 301-2.

18. Cheung V, Sirkin W. Listeriosis complicating pregnancy. CMAJ 2009; 181(11): 821-2.

19. Sánchez L, Capdevila E, Porta R, Molina V, Viñes L, Serra B. Listeriosis perinatal: también aumenta la incidencia en otras regiones. An Pediatr (Barc) 2010; 72: 149-50.

20. Tam C, Erebara A, Einarson A. Food-borne illnesses during pregnancy: prevention and treatment. Can Fam Physician 2010; 56 (4): 341-3.

21. Conly J, Johnston B. Listeria: A persistent food-borne pathogen. Can J Infect Dis Med Microbiol 2008; 19(5):327-8.

22. Ireton K. Entry of the bacterial pathogen Listeria monocytogenes in to mammalian cells. Cell Microbiol 2007; 9(6): 1365-75.

23. Chen S, Lu F, Lee P, Lu C, Chen C, Chou H, et al. Neonatal listeriosis. J Formos Med Assoc 2007; 106(2): 161-4.

Manuscrito recibido el: 16/08/2012

Aceptado para su publicación el: 10/12/2012

\section{Correspondencia:}

Nombre: Mg. Miguel Villegas Chiroque

Dirección: Jr. Puno 1002- Cercado de Lima, Perú

e-mail: mivichi2003@yahoo.es 